\title{
Foveal gravity: A robust illusion of color-location misbinding
}

\author{
Cristina R. Ceja ${ }^{1}$ (ID • Nicole L. Jardine ${ }^{1} \cdot$ Steven L. Franconeri ${ }^{1}$
}

Published online: 9 December 2019

(C) The Psychonomic Society, Inc. 2019

\begin{abstract}
Some types of object features, such as color, shape, or location, can be processed separately within the visual system, requiring that they be correctly "bound" to a single object via attentional selection of a subset of visual information. Forcing selection to spread too widely can cause an illusion where these features misbind to objects, creating illusory objects that were never present. Here, we present a novel display that produces a robust color-location misbinding illusion that we call foveal gravity (viewable at https://osf.io/2bndg/). When observers selected only a set of colored objects, colors were largely perceived in their correct locations. When observers additionally selected objects in the far periphery, colors in the near periphery migrated closer to the fovea on over $35 \%$ of trials. We speculate that foveal gravity occurs because locations closer to the fovea are more likely to defeat more peripheral locations in competitive interactions to "win" the task-relevant color.
\end{abstract}

Keywords Binding $\cdot$ Divided attention $\cdot$ Selective attention $\cdot$ Visual perception

Visual processing involves a division of labor, such that different features of objects are represented in a diverse set of neurophysiological regions, with color, shape, and location information computed independently in many cases (Hubel \& Wiesel, 1959; Livingstone \& Hubel, 1988; Zeki, 1973). Frequently occurring and familiar objects may be encoded by conjunctive representations of prebound features, represented by preexisting neural connections (VanRullen, 2009). As this processing is preattentive and automatic, it can often occur without attentional selection to bind together the features. However, it is unclear how a visual system could contain existing representations of any possible combination of object features that we might need to recognize in the world (Hummel \& Biederman, 1992). These object features must, instead, be processed in separate pathways, requiring that the features be later reintegrated, or "bound," back into a single object through the use of some form of attentional selection.

Decades of research have suggested that spatial attention is the critical mechanism for accurate binding in instances when prebound features are not available (e.g., Holcombe, 2009; Treisman \& Gelade, 1980). In particular, Treisman and

Cristina R. Ceja

crceja@u.northwestern.edu

1 Department of Psychology, Northwestern University, 2029 Sheridan Road, Cresap Hall 112, Evanston, IL 60208, USA
Gelade (1980) argued that even when a feature can be detected rapidly and in parallel across the visual field, binding that feature to a specific location requires focal attention. If true, then forcing selection to spread too widely across multiple objects should cause the features of those objects to misbind, such that an observer would see the wrong features attached to the wrong objects. Validating this prediction, many studies have found that when attention is spread across many objects in a briefly flashed display, observers perceive feature combinations that were never actually present (Cohen \& Ivry, 1989; Prinzmetal, Presti, \& Posner, 1986; Treisman \& Gelade, 1980; Treisman \& Schmidt, 1982; for review, see Franconeri, 2013; Prinzmetal, 2012).

Exploring the conditions under which these illusory combinations do and do not occur allows inferences about how the visual system combines these separate sources of object properties. Objects that are closer together are more likely to swap their surface features (Cohen \& Ivry, 1989; Lasaga \& Hecht, 1991), suggesting denser interconnections between nearby objects. Objects of similar colors can produce the perception of a blending of their colors, whereas objects of dissimilar colors can be perceived as having colors that are even more dissimilar (Golomb, 2015). These attraction and repulsion effects in color space occur when objects are spaced far apart (separated by 7 degrees visual angle), suggesting that the interconnections between representations of colors can mutually excite or inhibit one another, even at a distance. 
Previous demonstrations of spatially systematic misbindings have also found that information about bindings travels predominantly in the foveal-to-peripheral direction. Wu, Kanai, and Shimojo (2004) manipulated color and motion bindings within displays of moving dots (i.e., red dots moving up, among green dots moving down) with one color-motion conjunction at the fovea and the opposite color-motion conjunction in the periphery. These conjunctions were perceived veridically in the foveal region, but misbindings occurred in peripheral regions that contained the opposite conjunction: Observers who were shown an upward motion of red dots and a downward motion of green dots in the fovea reported seeing an identical binding of color and motion in the fovea and periphery, even though the pattern of color-motion in the periphery was opposite to that at fovea. Wu et al. (2004) proposed that foveal information is higher in precision, resulting in the foveal information outcompeting and overwriting the more peripheral information. A similar pattern of results has been found with color and orientation bindings (i.e., red horizontal, green vertical), with information bound at the fovea spreading outward to resolve binding in the periphery, even for task-irrelevant objects (Suzuki, Wolfe, Horowitz, \& Noguchi, 2013).

This work demonstrates a "filling-in" that progresses in a foveal-toward-peripheral direction: When feature bindings of color-orientation or color-motion in the periphery are in conflict with bindings in the fovea, perception in the periphery was dominated by perception in the fovea. However, this effect only arose when the feature-feature bindings in the fovea were present and consistent: When these foveal bindings were absent (Wu et al., 2004) or were mixed combinations of possible bindings (Suzuki et al., 2013), the peripheral filling-in disappeared. Suzuki et al. (2013) proposed that the visual system conducts a correlational visual analysis between features to aid in this foveal-toward-peripheral filling-in process, and uses only consistent feature bindings to resolve any ambiguity. But, note that this mechanism relies on accurate bindings of colors, orientations, and their locations within the fovea.

Here, we present a paradigm that produces a novel and robust misbinding illusion of colors to inaccurate locations in the opposite parafoveal-to-foveal direction under divided attention that we call foveal gravity. In contrast to previous work, object colors near the fovea were perceived in their veridical locations, whereas object colors outside of the fovea were highly likely to move inward, being misbound to and filling-in object locations closer to the fovea.

\section{Experiment 1}

In Experiment 1, we tested the robustness and directionality of color-location misbindings using sparse stimulus displays, consisting of central, colored diamonds and peripheral shapes (see Fig. 1).

\section{Method}

Participants Twenty participants (18-30 years old) completed the experiment. All participants had normal or corrected-tonormal vision, were given credit for participation, and gave informed written consent.

Apparatus The experiments were controlled by a MacOS computer running MATLAB. The display was presented on a CRT monitor with a $60-\mathrm{Hz}$ refresh rate, and $1,024 \times 768$ pixel resolution.

Stimuli The displays consisted of central and peripheral objects at fixed locations. Central objects were always diamonds horizontally spaced on either side of fixation (two within fovea and two within parafovea), with edges 1.0 degrees visual angle (dva) in length, and centroids 2.8 dva apart. Two diamonds were gray, and the two task-relevant diamonds were colored (green/green, red/red, red/green, or green/red). There was one task-relevant colored diamond located in each of the left and right visual hemifields (i.e., separate-hemifield locations; one on the left and one on the right of fixation), with one at the foveal location and the other at the parafoveal location (i.e., in the first and third positions, or in the second and fourth positions; they were never simultaneously presented only in the foveal positions or only in the parafoveal positions).

The six peripheral objects were white squares (edges $1.4 \mathrm{dva}$ ) and/or white circles (radius $0.85 \mathrm{dva}$ ). They were arranged radially from fixation, with three objects approximately 11.3 dva from fixation in each of the left and right visual hemifields. The peripheral objects could all be the same shape (three squares each in the left and right visual hemifields), or contain different shapes (two squares and one circle each mirrored in the left and right visual hemifields).

Procedure Trials began with a 400-ms central fixation cross, where participants were instructed to keep their eyes during the trial. A premask of four horizontal gray diamonds was displayed for $800 \mathrm{~ms}$. This was followed by the main display (80 ms), which still included the fixation cross and four horizontal diamonds; however, now two of the gray diamonds had changed to any combination of red and/or green. Simultaneously, six white objects (i.e., six squares, or four squares and two circles) also appeared in the periphery. This trial display was followed by a postmask of four gray diamonds and a fixation cross for $800 \mathrm{~ms}$, followed by only a fixation cross for $300 \mathrm{~ms}$. 


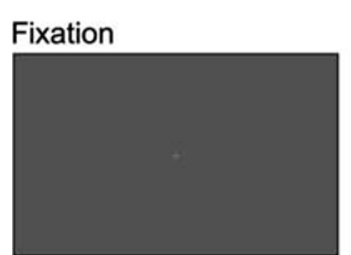

$400 \mathrm{~ms}$

\section{Pre-Mask}

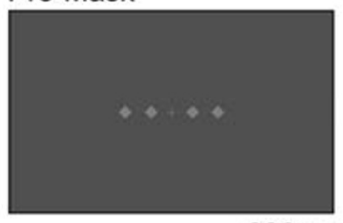

$800 \mathrm{~ms}$

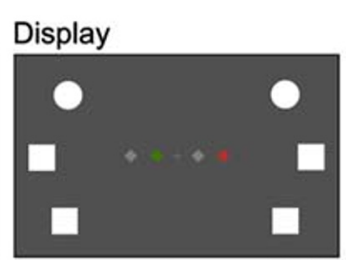

$80 \mathrm{~ms}$
Post-Mask

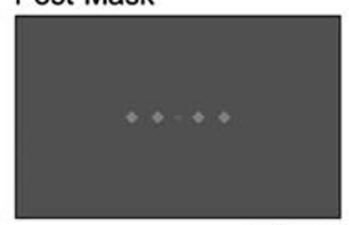

End of Trial

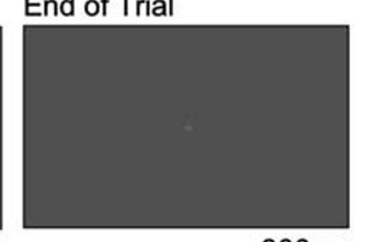

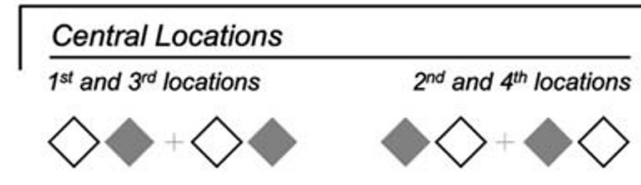

b Response Procedure

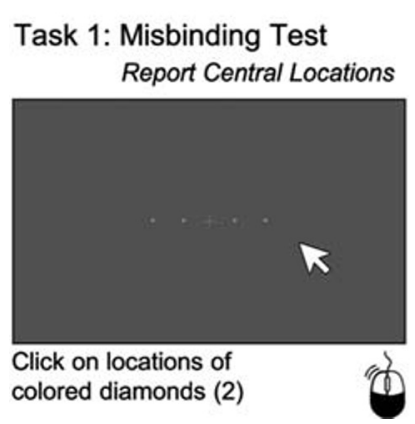

Fig. 1 Procedure for Experiment 1 (displays are to scale). a Display procedure: Participants saw displays of central objects that varied in their locations and colors, and peripheral objects that varied in shape. $\mathbf{b}$ Response procedure: Participants reported the locations of the colored

Participants then viewed the response screens for the three tests: misbinding test (of central object color-location bindings), a feature perception test (of central object colors), and a divided attention test (of peripheral object shapes). Participants performed all three tests after each trial in this order, with the misbinding test performed first to minimize the chance that any mislocalizations were due to failures in memory.

For the misbinding test, participants reported the locations of the task-relevant colored diamonds. Participants viewed a response screen of four gray placeholder dots, with a "Clear" button to clear previous mouse clicks, and a "Done" option to advance to the next task. Participants were told that the four gray placeholder dots were references to where all of the diamonds appeared, but that they should click anywhere on the screen where they saw the colored diamonds, specifically. To select their responses, participants used the mouse to click each of the two locations where they saw colored diamonds appear, with each click of the mouse generating a white diamond in the location of the mouse click.

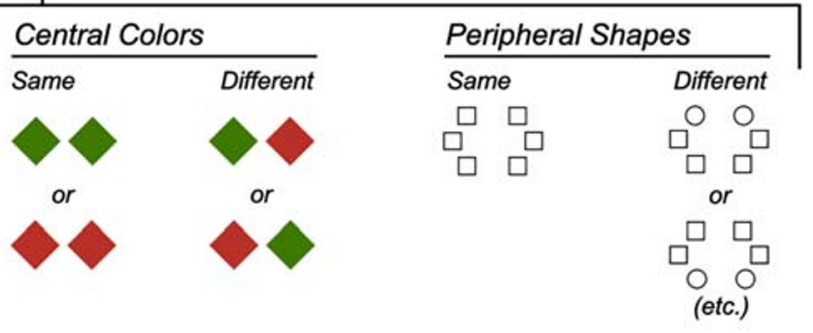

$300 \mathrm{~ms}$

Task 2: Feature Perception Test

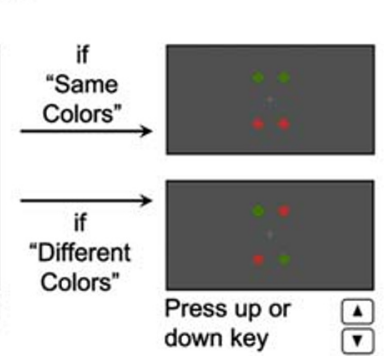

Task 3: Divided Attention Test Report Peripheral Shapes

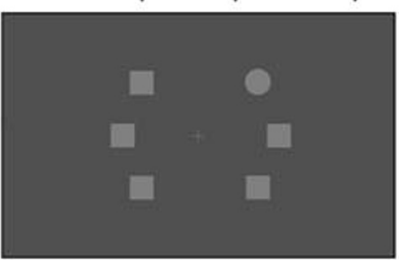

Press left ("Same") or right ("Different") key

central objects ("Task 1: Misbinding Test"), the colors of the central objects ("Task 2: Feature Perception Test"), and then reported whether the peripheral objects were all the same or different shapes ("Task 3: Divided Attention Test"). (Color figure online)

Next, for the feature perception test, participants reported whether the colors were the same or different between the two task-relevant colored diamonds (same-different color task), followed by specifying their exact colors (specific-color task). For the same-different color task, participants viewed a screen with text that read "Same Colors" in the left visual hemifield and "Different Colors" in the right visual hemifield. Participants were instructed to indicate whether the two colored diamonds they saw during the trial were the same color (i.e., both red or both green) or different colors (i.e., one red and one green) by using the left or right arrow keys. This was immediately followed by a specific-color task depending on their response. If "Same Colors" had been selected for the same-different color task, participants then saw a response screen containing a fixation cross, a pair of two green horizontal diamonds located in the top visual hemifield (above the fixation cross), and a pair of two red horizontal diamonds located in the bottom visual hemifield (below the fixation cross) for the specific-color task. In contrast, if "Different Colors" was selected for the same-different color task, participants viewed a response screen containing a green diamond 
to the left of a red diamond in the top visual hemifield, and a red diamond to the left of a green diamond located in the bottom visual hemifield. To select their answer for the specific-color task, participants used the up or down arrow keys.

Lastly, for the divided attention test, participants reported whether the three peripheral shapes each located in left and right visual hemifields were all squares or a combination of squares and circles. Participants were shown a screen with a fixation cross, three vertical gray squares located in the left visual hemifield (indicating that they saw all squares in the periphery), and a vertical column of two gray squares with one gray circle located in the right visual hemifield (indicating that they saw squares and circles in the periphery). To select a response, participants used the left or right arrow keys. This response then initiated the next trial.

Each participant was tested in four blocks of 24 trials, for a total of 96 trials. Participants were given a 60 -second break between each block. Trials between blocks were randomized, and all variables were balanced throughout the experiment (i.e., all combinations of central colors, locations, and peripheral shapes were equally likely to occur during the experiment). As such, half of all trials consisted of same-color diamonds, and half of different-color diamonds; half of all trials also displayed all squares for the peripheral objects, and half displayed squares and circles.

Analysis Participants with an average performance lower than $70 \%$ accuracy for the divided attention test and for the samedifferent color task were excluded from further analysis $(N=$ $20-2=18$ ). For any participants with a low accuracy for these tests below the cutoffs, it is uncertain whether they were actively dividing attention to the peripheral shapes (shown through performance on the divided attention test) while still attending to the central diamonds (shown through performance on the same-different color task), so these participants were excluded from analysis. An alternative exclusion criterion of two standard deviations below the mean for the divided attention test (at $65.6 \%$ accuracy) and the same-different color task (at $70.3 \%$ accuracy) resulted in the same participants being excluded from further analysis. Additionally, as diamonds were 1.4 dva in width, single trials in which distances between clicked and true locations were larger than 1.4 degrees visual angle were excluded as noise (16 trials, or fewer than $0.01 \%$ of trials; $97.97 \%$ of clicks were within 1 dva of a diamond centroid).

Average accuracy was first analyzed for the divided attention test, and only trials with accurate responses for this test were analyzed for the same-different color task. Of those trials with both accurate divided attention test and same-different color task responses, responses from the specific-color task were then averaged for accuracy, and responses for the misbinding test were analyzed. All trials, same-color and different-color diamonds, were included in these analyses as indicators of overall performance.

\section{Results}

The results showed that participants were able to divide attention between peripheral and central objects. Errors were low for the divided attention test of peripheral shape reports (14.4\%) and for same-different color reports for central objects $(11.2 \%)$. Feature perception was also accurate. For the specific-color task, feature errors of color identification occurred only on $6.6 \%$ of trials, suggesting observers veridically perceived the colors in the display.

But color-location misbindings, where participants mislocalized these accurately perceived colors, occurred on $36.1 \%$ of divided attention trials (35.2\% of same-color trials; $37.0 \%$ of different-color trials). Colored diamonds that were mislocalized ( $36.1 \%$ of all trials) were far more likely to be reported at the object location that was closer to fixation $(93.8 \%$ of mislocalized trials) rather than away from fixation $(1.1 \%$ of mislocalized trials). ${ }^{1,2}$ As such, we call this effect foveal gravity: Object colors were perceived accurately, but were localized closer to the fovea, ${ }^{3}$ under divided attention.

\section{Experiment 2}

To claim that divided attention produced these color-location misbindings, these illusory misbindings should be reduced when attention is focused on the central objects alone. In Experiment 2, participants performed similar tests to Experiment 1 under conditions of divided attention (i.e., attention is divided between central and peripheral objects), and under focal attention (i.e., attention is focused only on central objects).

\footnotetext{
${ }^{1}$ The remaining $5.0 \%$ of mislocalized trials included both colored diamonds being reported at both locations where uncolored diamonds appeared (one closer to fixation and one away from fixation).

${ }^{2}$ Without excluding trials that were incorrect in the divided attention test, color-location misbindings occurred on $35.5 \%$ of trials (34.9\% of samecolor trials; $36.1 \%$ of different-color trials). Mislocalized colored diamonds were more likely to be reported closer to fixation $(98.4 \%)$ rather than farther from fixation $(1.6 \%)$. The remaining $1.6 \%$ of trials included both colored diamonds being reported at both locations where uncolored diamonds appeared.

${ }^{3}$ Feature swaps between two objects can occur in misbinding illusions, but were less frequent in this paradigm. In an independent analysis conducted only on the 680 different-color trials, we looked at color feature swaps (e.g., reporting red-green instead of green-red). When both locations were correct (62.21\% of trials), color swaps occurred on $7.80 \%$ of these trials. When at least one location was incorrect (37.79\% of trials), color swaps occurred on $15.18 \%$ of these trials.
} 


\section{Method}

Eighteen new participants (18-35 years old) completed Experiment 2. For both the divided and focal attention conditions, we used a similar paradigm to Experiment 1, with a few modifications.

Experiment 2 added both-foveal (i.e., central colored objects in the second and third positions) and both-parafoveal (i.e., central colored objects in the first and fourth positions) displays to the divided and focal attention conditions. This change was made to account for a concern that now that participants were no longer dividing attention across the display in the focal attention condition, observers would notice regularities in the locations of the central objects (i.e., task-relevant colored objects always appeared in the first and third positions, or in the second and fourth positions). This knowledge could then contaminate guessing for the feature and location reports. Therefore, colored diamonds were now free to occupy any combination of foveal or parafoveal locations in both conditions, though still restricted to one colored object in each of the left and right visual hemifields.

In the divided attention condition, a premask was displayed, consisting of four gray squares (1.0 dva edge length). This was followed by the main display consisting of a fixation cross, four central objects that were always squares, and four white peripheral objects which could all be vertically concave squares or all vertically convex squares (two located in each of the left and right visual hemifields). Two of the central squares were displayed as any combination of red and/or green, and could appear in any combination of separate-hemifield locations (i.e., first and third, first and fourth, second and third, or second and fourth positions). The remaining two central squares were shown in gray. This trial display was then followed by a postmask of four gray squares and a fixation cross. Participants were instructed to report the locations and colors of the central colored squares, and the shape identities of the peripheral objects.

In the focal attention condition, observers viewed an identical premask to the divided attention blocks. They then viewed a main display with a fixation cross, four central objects that varied in shape to all be vertically concave squares or all vertically convex squares, and four peripheral objects which were always white squares (two located in each of the visual hemifields). Two of these central objects were displayed red and/or green and could appear in any separatehemifield locations (i.e., first and third, first and fourth, second and third, or second and fourth positions), while the remaining central objects were shown in gray. This display was again followed by a postmask of four gray squares and a fixation cross. Participants were instructed to ignore the peripheral objects, and report the locations and colors of the central colored objects, and the shape identities of the central objects.
Similar to Experiment 1, participants again performed a misbinding test (of central object color-location bindings), a feature perception test (of central object colors), and a divided or focal attention test (of peripheral or central object shape) depending on the condition (i.e., divided attention condition or focal attention condition, respectively). All three tests were performed at the end of each trial in the order listed.

For the divided or focal attention conditions, response screens were identical to Experiment 1 for the misbinding test and the feature perception test (with the modification that any diamonds shown during either of the response screens were now squares). For the divided or focal attention tests, response screens were modified so that participants now indicated whether the peripheral or central squares were all concave or convex. Specifically, for the divided attention condition, the divided attention test probed whether the peripheral shapes were concave or convex squares. Participants viewed a response screen with a fixation cross, two gray vertically concave squares located in the left visual hemifield, and two gray vertically convex squares located in the right visual hemifield. Participants used the left or right arrow keys to indicate whether the peripheral squares were all concave squares or all convex squares, respectively. For the focal attention condition, participants reported whether the central shapes were all concave or convex squares by using the left or right arrow keys on a screen with a fixation cross, one gray concave square located in the left visual hemifield, and one gray convex square located in the right visual hemifield.

The number of decisions in both divided and focal attention conditions were equated to two decisions about colors (i.e., same-different color task and specific-color task), two about locations (i.e., clicking each of the two locations where the colored objects appeared), and one about shapes (i.e., divided or focal attention test).

Each participant was tested in eight blocks (with four blocks each for the divided attention and focal attention conditions) of 16 trials each, for a total of 128 trials. There was a 60-s break between each block. Before each block, participants were cued with a screen of task instruction text. Divided attention block instructions were to "Mainly focus on detecting the white shapes on the sides. Try your best to attend to the center." Focal attention block instructions were to "Focus ONLY on the center, and mainly on detecting shapes. IGNORE the white shapes on the sides." Condition blocks alternated, and the first task was counterbalanced.

Analysis Participants with an average accuracy performance lower than $70 \%$ for the divided or focal attention tests of shapes and for the same-different color task in the divided and focal attention conditions were excluded from further analysis $(N=18-5=13)$. An alternative exclusion criterion of two standard deviations below the mean for the divided and focal attention tests of shapes (at 52.9\% accuracy and at 
$54.6 \%$ accuracy, respectively) and for the same-different color task (at $60 \%$ accuracy and at $83.1 \%$ accuracy for divided and focal attention conditions, respectively) resulted in the same participants being excluded from further analysis.

For both the divided and focal attention conditions, only trials in which the colored shapes appeared in the first and third locations or in the second and fourth locations were analyzed, so as to be comparable to Experiment 1. Inferential statistics were conducted with planned paired $t$ tests, with effect sizes reported as Cohen's $d$.

\section{Results}

Feature perception was accurate overall under divided and focal attention conditions. Focal attention tests of central shapes and divided attention tests of peripheral shapes were of equally low errors $(17.2 \%$ and $16.4 \%$, respectively), $t(12)=0.24, p=.814$, Cohen's $d=0.11$. Observers were also able to accurately perceive colors with low error rates under divided and focal attention. Feature errors of color identification in the feature perception test occurred on only $2.7 \%$ of focal attention trials and on $5.5 \%$ of divided attention trials, $t(12)=1.02, p=.326, d=0.34$. Therefore, if any significant difference in color-location misbindings (the correct-colored object reported at the wrong

\section{Divided Attention Condition}

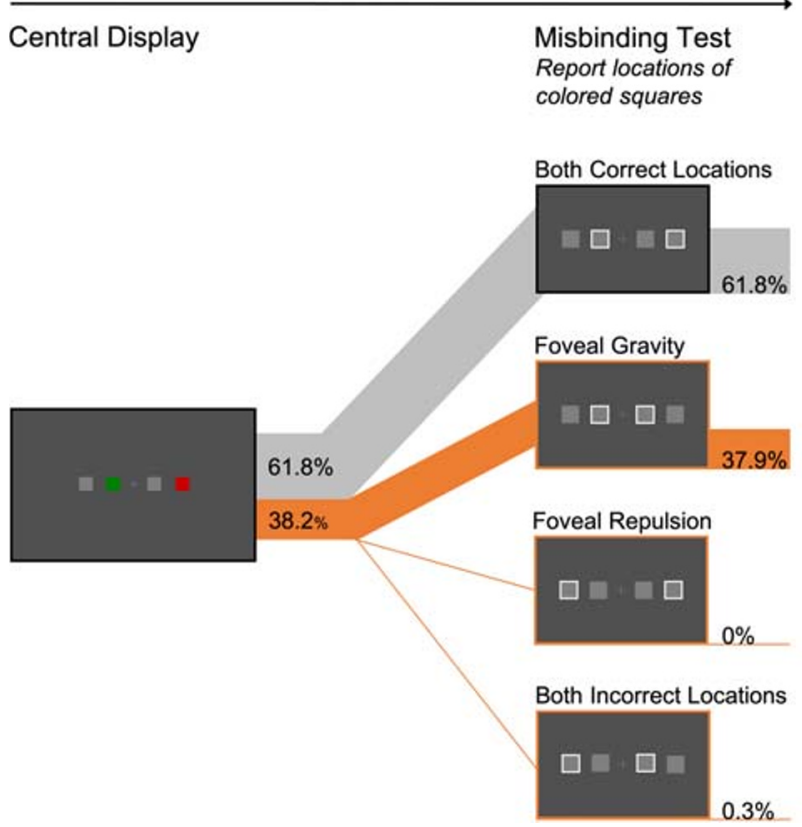

Fig. 2 Results from Experiment 2 for the misbinding test in the divided attention and focal attention conditions. The first and fourth columns ("Central Display") are an example of central colored objects that a participant may have seen during a trial (e.g., a red and a green object at the second and fourth locations, respectively; display is not to scale, and peripheral objects are not depicted in this figure). The second and third columns ("Misbinding Test") are location reports for these colored objects, indicating the percentage of overall trials in which participants were correct in localizing the objects ("Both Correct Locations"), when location) is found between the divided and focal attention blocks, it should be because veridical color-location binding is selectively hindered when attention is divided.

Under conditions of divided attention, as in Experiment 1, color-location misbindings occurred on $38.2 \%$ of trials (see Fig. 2). But under focal attention, these color-location misbindings occurred on only $7.1 \%$ of trials, $t(12)=4.90, p$ $=.0004, d=1.96$. Although participants were able to accurately perceive the objects' colors, they systematically and frequently failed to bind those colors to their respective locations during divided attention.

Foveal gravity was prevalent once more under divided attention: colored squares that were mislocalized (38.2\% of all trials) were drastically more likely to be reported closer to fixation (99.1\% of mislocalized trials) than farther away from fixation $(0.0 \%){ }^{4,5}$

\section{General discussion}

These experiments demonstrate that colors are accurately perceived but frequently bound to the wrong locations without focal attention. This finding is consistent with previous research indicating that focal attention is required for proper

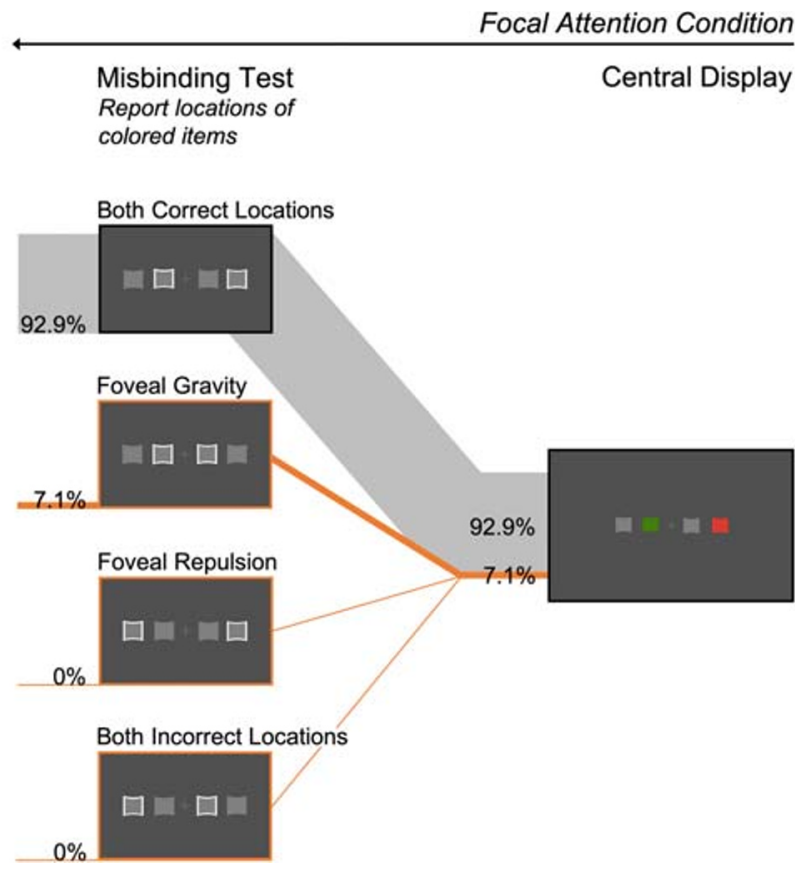

objects were localized closer to fixation ("Foveal Gravity"), when objects were localized away from fixation ("Foveal Repulsion"), or when participants were entirely incorrect in their choice of locations ("Both Incorrect Locations"). Percentages indicate what total percentage of trials participants responded in a given way to the misbinding test, given that they were accurate in both the divided attention test and the central feature perception test. Line thickness shows percentage of trials, with orange lines indicating color-location misbindings. (Color figure online) 
location binding of features (Luck \& Ford, 1998), and is consistent with the prediction that focal attention is, under some conditions, needed to bind object features to their locations (Treisman \& Gelade, 1980). Existing evidence also shows that precise localization of features - such as the high-precision localization needed in the present paradigm - requires focused attention on the feature location (Hyun, Woodman, \& Luck, 2009). In that experiment, observers detected a color-defined target among many other items and reported either its presence, its low-precision location (i.e., Was the target in the upper or lower visual hemifield?), or its high-precision location (i.e., Was the target above or below a reference line?). Neurophysiological indices of attention (N2pc) showed that even low-precision bindings demanded attentional selection, though high-precision reports demanded more sustained selection. The current results are also consistent with the idea that higher precision localization (discriminating within a visual hemifield) requires more focused and sustained attentional selection. Participants were able to locate one colored object in each visual hemifield (low-precision bindings), but performed poorly at specifically locating the colors within the hemifields (higher-precision bindings) when attentional selection was hindered in the divided attention condition. Yet when more focused selection was allowed in the focal attention condition, participants located the colored objects correctly.

The particularly sparse displays used here also allow the paradigm to more clearly demonstrate binding illusions, by minimizing the role of postperceptual memory confusion and related biases. Many previous studies used complex displays that included objects defined by many distinct feature values (i.e., items in two possible shapes, four possible colors, two possible sizes, and in two possible formats - outlined or filled - in Treisman \& Schmidt, 1982; six possible shapes and six possible colors in Treisman \& Zhang, 2006). In this past work, there were higher rates of feature misbindings (Cohen \& Ivry, 1989; Gibson, 1971; LaBerge, 1973; Treisman \& Schmidt, 1982; Treisman \& Zhang, 2006; Tsal, Meiran, \& Lavie, 1994), but also greater uncertainty about the feature identities themselves (Prinzmetal et al., 1986; Shaw, 1982). It is possible that this uncertainty in feature identification contributed to postperceptual confusion about what was seen at a postattentive or decision stage of processing (Johnston \& Pashler, 1990; Wolfe, Klempen, \& Dahlen, 2000). Put differently, previously reported rates of illusory misbindings may have been artificially inflated by decision confusion, rather than reflecting illusory perceptions per se. Demonstrating feature misbinding with sparser displays reduces the possibility of such inflation by decreasing the uncertainty surrounding the feature identities themselves.

While this current paradigm allows for a new demonstration of the important role for selective attention in feature binding, it also illustrates a novel directional pattern in misbinding: foveal gravity dominated in trials of divided attention, such that colors were overwhelmingly mislocated in a direction closer to fixation. The possibility exists that directional patterns like foveal gravity may have existed undiscovered in previous work on binding and attention (e.g., Treisman \& Schmidt, 1982).

But why does foveal gravity occur-why do the features become arbitrarily and consistently bound to locations closer to the fovea? Past research on spatial localization shows that features tend to be localized with a bias closer to the fovea. When asked to remember the location of a briefly flashed target and then report where it appeared, observers tended to report remembered locations as shifted toward fixation, and this bias was larger with a broader spatial distribution of attention compared with focused (e.g., Mateef \& Gourevich, 1983; Werner \& Diedrichsen, 2002). However, past foveal biases were relatively small in magnitude (e.g., targets at 4 5 degrees of eccentricity in the parafovea were shifted toward the fovea by 1 degree of visual angle in Mateef \& Gourevich, 1983, and in Fortenbaugh \& Robertson, 2011). In the current paradigm, foveal biases were found to be large: foveal gravity resulted in objects 4 dva in the periphery being shifted toward fovea by 2.8 dva.

Could landmarks in the display have a potential effect on foveal bias? In the current paradigm, landmarks (i.e., the four gray diamonds or squares, and the four gray placeholder dots) were present throughout the stimulus display and during the response phase, respectively. Observers' clicked locations overwhelmingly landed on these landmarks, rather than an intermediate location. However, in previous studies, the presence of landmarks in a display tended to reduce foveal bias, and could produce spatial averaging of shifts toward the landmark (Werner \& Diedrichsen, 2002). This spatial averaging between target and distractor object locations might have been due to how these landmarks affected the location of spatial attention, or due to representational averaging in memory (Kerzel, 2002).

A more likely theme in past work to explain the directionality of foveal gravity is that this bias might be due to asymmetrical distributions or weightings of population activity near the fovea (Bocianski, Müsseler, \& Erlhagen, 2010). Their model proposed that spatially tuned neurons have asymmetrical distributions within their interaction profiles, such that neurons closer to the fovea were more heavily weighted in a localization task. Uncertainty caused competition for a decisive spatial location for an object, and that competition was biased spatially toward the fovea. This bias occurred more strongly when the location was more uncertain, such as when attention was distributed. Focal attention increased certainty, which decreased the bias. The competition that caused this bias may have been restricted to the same visual hemifield, which tends to be more interconnected, relative to the lack of connection between hemifields (Franconeri, Alvarez, \& Cavanagh, 2013). Indeed, the current paradigm did not 
produce color mislocalizations that crossed the horizontal midline which separates the left and right visual hemifields. Thus, the selection of a spatial location near the fovea might "pull" a weak signal from a peripheral object because of the heavier interconnections within a hemifield, but not across to the other hemifield.

The present work demonstrates a high prevalence ( $\sim 35 \%$ of trials) of visual features shifting in a parafoveal-toward-foveal direction to new object locations. But are larger location shifts of features possible? Can these shifts cross a visual hemifield or quadrant boundary? Can biasing competition for "ownership" of the unbound color be manipulated by biasing competition to win its location, perhaps with an attentioncapturing flash at the right moment?

Acknowledgements We thank Caitlyn McColeman and Elsie Lee for helpful comments.

Open practices statement The data for all experiments are available at https://osf.io/2bndg/, and none of the experiments were preregistered.

Author contributions S. L. Franconeri developed the study concept. C. R. Ceja and S. L. Franconeri contributed to the study design. Testing and data collection were performed by C. R. Ceja. C. R. Ceja performed the data analysis and interpretation under the supervision of S. L. Franconeri and N. L. Jardine. C. R. Ceja and N. L. Jardine drafted the manuscript, and S. L. Franconeri provided critical revisions. All authors approved the final version of the manuscript for submission.

\section{References}

Bocianski, D., Müsseler, J., \& Erlhagen, W. (2010). Effects of attention on a relative mislocalization with successively presented stimuli. Vision Research, 50(18), 1793-1802.

Cohen, A., \& Ivry, R. (1989). Illusory conjunctions inside and outside the focus of attention. Journal of Experimental Psychology: Human Perception and Performance, 15(4), 650-663.

Fortenbaugh, F. C., \& Robertson, L. C. (2011). When here becomes there: Attentional modulation of foveal bias in peripheral localization. Attention, Perception, \& Psychophysics, 73(3), 809-828.

Franconeri, S. L. (2013). The nature and status of visual resources. In D. Reisberg (Ed.), Oxford handbook of cognitive psychology. Oxford, UK: Oxford University Press. https://doi.org/10.1093/oxfordhb/ 9780195376746.013.0010

Franconeri, S. L., Alvarez, G. A., \& Cavanagh, P. (2013). Flexible cognitive resources: Competitive content maps for attention and memory. Trends in Cognitive Sciences, 17(3), 134-141.

Gibson, E. J. (1971). Perceptual learning and the theory of word perception. Cognitive Psychology, 2(4), 351-368.

Golomb, J. D. (2015). Divided spatial attention and feature-mixing errors. Attention, Perception, \& Psychophysics, 77, 2562-2569.

Holcombe, A. O. (2009). Seeing slow and seeing fast: Two limits on perception. Trends in Cognitive Sciences, 13(5), 216-221.

Hubel, D. H., \& Wiesel, T. N. (1959). Receptive fields of single neurones in the cat's striate cortex. The Journal of Physiology, 148, 574-591.
Hummel, J. E., \& Biederman, I. (1992). Dynamic binding in a neural network for shape recognition. Psychological Review, 99(3), 480 517.

Hyun, J.-S., Woodman, G. F., \& Luck, S. J. (2009). The role of attention in the binding of surface features to locations. Visual Cognition, 17(1/2), 10-24.

Johnston, J. C., \& Pashler, H. (1990). Close binding of identity and location in visual feature perception. Journal of Experimental Psychology: Human Perception and Performance, 16(4), 843-856.

Kerzel, D. (2002). Memory for the position of stationary objects: Disentangling foveal bias and memory averaging. Vision Research, 42(2), 159-167.

LaBerge, D. (1973). Attention and the measurement of perceptual learning. Memory \& Cognition, 1(3), 268-276.

Lasaga, M. I., \& Hecht, H. (1991). Integration of local features as a function of global goodness and spacing. Perception \& Psychophysics, 49(3), 201-211.

Livingstone, M., \& Hubel, D. (1988). Segregation of form, color, movement, and depth: Anatomy, physiology, and perception. Science, 240(4853), 740-749.

Luck, S. J., \& Ford, M. A. (1998). On the role of selective attention in visual perception. Proceedings of the National Academy of Sciences of the United States of America, 95, 825-830.

Mateef, S., \& Gourevich, A. (1983). Peripheral vision and perceived visual directions. Biological Cybernetics, 49, 111-118.

Prinzmetal, W. (2012). At the core of feature integration theory: On Treisman and Schmidt (1982). In J. Wolfe \& L. Robertson (Eds.), From perception to consciousness (pp. 211-216). New York, NY: Oxford University Press.

Prinzmetal, W., Presti, D. E., \& Posner, M. I. (1986). Does attention affect visual feature integration? Journal of Experimental Psychology: Human Perception and Performance, 12(3), 361-369.

Shaw, M. L. (1982). Attending to multiple sources of information: I. The integration of information in decision making. Cognitive Psychology, 14(3), 353-409.

Suzuki, M., Wolfe, J. M., Horowitz, T. S., \& Noguchi, Y. (2013). Apparent color-orientation bindings in the periphery can be influenced by feature binding in central vision. Vision Research, 82, 5865.

Treisman, A. M., \& Gelade, G. (1980). A feature-integration theory of attention. Cognitive Psychology, 12(1), 97-136.

Treisman, A., \& Schmidt, H. (1982). Illusory conjunctions in the perception of objects. Cognitive Psychology, 14, 107-141.

Treisman, A., \& Zhang, W. (2006). Location and binding in visual working memory. Memory \& Cognition, 34(8), 1704-1719.

Tsal, Y., Meiran, N., \& Lavie, N. (1994). The role of attention in illusory conjunctions. Perception \& Psychophysics, 55(3), 350-358.

VanRullen, R. (2009). Binding hardwired versus on-demand feature conjunctions. Visual Cognition, 17(1/2), 103-119.

Werner, S., \& Diedrichsen, J. (2002). The time course of spatial memory distortions. Memory \& Cognition, 30(5), 718-730.

Wolfe, J. M., Klempen, N., \& Dahlen, K. (2000). Postattentive vision. Journal of Experimental Psychology: Human Perception and Performance, 26(2), 693-716.

Wu, D. A., Kanai, R., \& Shimojo, S. (2004). Steady-state misbinding of colour and motion. Nature, 429, 262.

Zeki, S. M. (1973). Colour coding in rhesus monkey prestriate cortex. Brain Research, 53, 422-427.

Publisher's note Springer Nature remains neutral with regard to jurisdictional claims in published maps and institutional affiliations. 